\title{
On the driving force for crack growth during thermal actuation of shape memory alloys
}

\author{
T. Baxevanis*, A.F. Parrinello ${ }^{1}$, D.C. Lagoudas \\ Department of Aerospace Engineering, Texas A $6 M$ University, College Station, TX 77843
}

\begin{abstract}
The effect of thermomechanically-induced phase transformation on the driving force for crack growth in polycrystalline shape memory alloys is analyzed in an infinite center-cracked plate subjected to a thermal actuation cycle under mechanical load in plain strain. Finite element calculations are carried out to determine the mechanical fields near the static crack and the crack-tip energy release rate using the virtual crack closure technique. A substantial increase of the energy release rate-an order of magnitude for some material systems-is observed during the thermal cycle due to the stress redistribution induced by large scale phase transformation. Thus, phase transformation occurring due to thermal variations under mechanical load may result in crack growth if the crack-tip energy release rate reaches a material specific critical value.
\end{abstract}

Keywords: Shape Memory Alloys, Actuation, Phase Transformation, Fracture toughness, Finite elements

\section{Introduction}

Shape Memory Alloys (SMAs) are metallic materials that can recover large, apparently permanent, strains when subjected to the appropriate thermal procedure. Generally, these materials can be plastically deformed at some relatively low temperature, and upon exposure to some higher temperature, they return to their shape prior to deformation. The physical mechanism that drives this shape recovery is a reversible diffussionless solid to solid transformation from austenite to martensite and vice versa. SMA actuators take advantage of this property to provide a significant amount of actuation with an extremely small

\footnotetext{
${ }^{*}$ Corresponding author

Email addresses: theocharis@tamu.edu (T. Baxevanis), antoninofranc.parrinello@gmail.com (A.F. Parrinello), lagoudas@tamu.edu (D.C. Lagoudas)

${ }^{1}$ Present address: Department of Engineering Science, University of Oxford, Oxford, OX1 3PJ
}

Preprint submitted to Elsevier

November 18, 2015

(C) 2016. This manuscript version is made available under the Elsevier user license http://www.elsevier.com/open-access/userlicense/1.0/ 


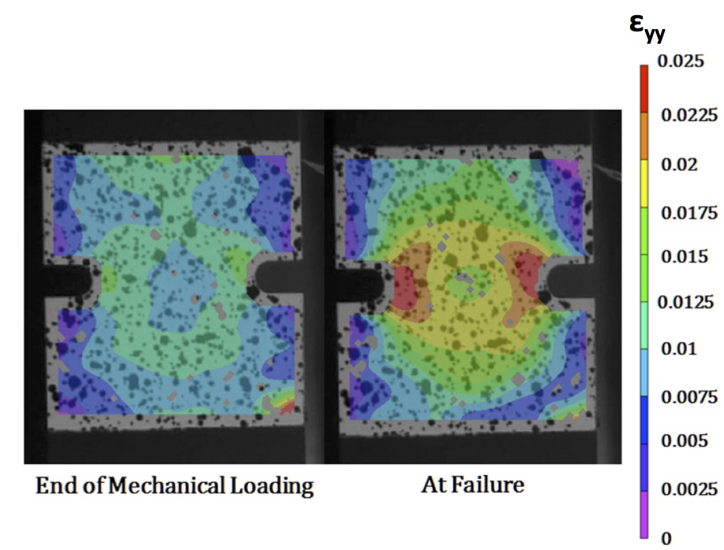

Figure 1: Strain in the direction of the tensile applied loading (vertical to the axis of the notches) at the end of the isothermal loading (60\% of the isothermal strength at that temperature) and just before the formation of an unstable crack under the same bias load at a lower temperature, higher than the martensitic-finish temperature. Taken from [14].

envelope volume. SMA actuators are therefore used as an alternative to electromagnetic actuators when a small volume and/or large force and stroke are required and thermodynamic efficiency is not essential [1-3].

The effective implementation of SMAs as actuators requires the assessment of their fracture properties under actuation loading paths, i.e., under combined thermal and mechanical loading. Such studies will enable the practice of fracture mechanics concepts in SMA actuators, and can potentially result in criteria that will connect fracture properties to measurable and hopefully controllable thermomechanical characteristics and microstructural features to provide a knowledge base that can be used in the design of SMA material systems. However, to the best of the authors' knowledge, such studies are lacking in literature. As recently observed, notched, precipitation-hardened $\mathrm{Ni}_{60} \mathrm{Ti}_{40}$ (wt\%) SMA specimens may fail during cooling under a constant applied tensile load that is lower than the isothermal strength at the beginning of cooling. For the U-shape notched specimens tested ${ }^{2}$, failure by the formation of an unstable crack during cooling was observed for bias load levels as low as $60 \%$ of the isothermal load needed for failure at the beginning of cooling (Figure 1). This is an intriguing response, characteristic of SMAs, that from an energetic point of view may seem in disagreement with the general view of dissipative processes resulting in an enhancement of fracture toughness. In most conventional elastic-plastic metals, cooling under a constant load does not considerably affect the existing plastic zone close to the crack tip and thermal contraction results in crack shielding

\footnotetext{
${ }^{2}$ The experiments were performed at Texas A\&M University and at Naval Research Laboratory and will be presented elsewhere.
} 
rather than anti-shielding.

Currently, only the fracture response of SMAs during mechanical loading at constant ambient temperatures has received attention in the literature [414]. These studies have been driven by the desire to understand the effect of stress-induced phase transformation or detwinning on the fracture properties of SMAs, stemming mainly from their suitability for medical applications. In their finite element analysis of SMAs obeying power-law hardening flow theory for the evolution equations of the plastic strains, Baxevanis et al. [10] arrived at the conclusion that stress redistribution due to stress-induced martensitic transformation results in plastic zone sizes at the vicinity of the crack tip an order of magnitude smaller that the size expected in conventional elastic-plastic materials. Experimental evidence on binary martensitic $\mathrm{Ni}_{50.3} \mathrm{Ti}_{49.7}($ at\%) and pseudoelastic $\mathrm{Ni}_{50.7} \mathrm{Ti}_{49.3}$ (at\%) material systems that show appreciable plastic deformation in uniaxial loading $[7,15]$, indicating that phase transformation reduces the extent of plastic deformation close to the crack tip, support the numerical results. In [15], cleavage fracture and river markings consistent with markings from traditional brittle intermetallic alloys were reported in $\mathrm{Ni}_{50.7} \mathrm{Ti}_{49.3}$ systems containing semi-coherent precipitates. Gollerthan et al. [7] observed that the crack tips stay sharp during crack growth in both the aforementioned martensitic and pseudoelastic SMAs alloys and all martensitic features in front of the crack tip in the pseudoelastic ones disappear after unloading. The latter observation excludes the formation of stabilized martensite, which cannot reverse transform, because it is hindered by the presence of dislocations. Crack tip blunting is associated with dislocation processes, and the absence of blunting in NiTi may well reflect the fact that dislocation activity at the crack tip plays a much smaller role. Therefore it seems reasonable to assume that in SMAs, even beyond those that show appreciable strength against dislocation plasticity such as, for example, nano-precipitation hardened SMAs, the length scale of the plastic deformation zone surrounding the crack tip is small enough to ensure the validity of (i) an analysis of the fracture response of SMAs on the basis of a constitutive law that does not account for plastic deformation, and (ii) a single parameter for characterizing the fracture toughness of martensite forming at the crack tip. Thus, crack growth can be presumed to proceed at a critical level of the crack-tip energy release rate, which is considered to be a constant material property. However, more experiments are needed to clarify the extent of plastic deformation near crack tips and its importance on the fracture toughness of SMAs.

All of the published data on the fracture toughness values of SMA materials is based on the premise of linear elastic fracture mechanics (LEFM) and ASTM standards for conventional elastic-plastic materials. Most of this data, as discussed in recent review papers [14, 16], is questionable in the temperature range in which the SMA materials are martensitic or pseudoelastic. The reason being that ASTM standards should be interpreted as to require the zone of non-linear deformation close to the crack tip, regardless of the mechanism, to be smaller than a fraction of all the characteristic dimensions of the crack configuration. With respect to SMAs, the latter restriction leads to a requirement on the size of 
the stress-induced transformation/detwinning zone, and not just on the plastic zone size, being small enough for LEFM to be valid. For the latter requirement to be satisfied, the SMA specimens may be prohibitively large depending on the ambient temperature. An alternative may be to measure the fracture toughness using the $J$-integral as a fracture criterion, for which the requirements on specimen sizes are much less strict than those for a valid $K_{I_{c}}$-value. For temperatures higher than $M_{d}$, the austenitic phase is stable irrespectively of the load levels, the resulting stress-strain curve is similar to that of a conventional metal, and thus the published data in $[7,18,19]$ is representative of the fracture toughness value of austenite.

The crack-tip stress-induced phase transformation is primarily responsible for the phenomenon of stable crack growth in SMAs under monotonically increasing load or displacement conditions [20]. This toughening effect of stressinduced phase transformation is associated with the energy dissipated by the transformed material left behind the advancing crack tip [11, 21-24]. Reverse phase transformation that may occur due to unloading in the wake of the growing crack distinguishes the toughening response of SMAs from that of other dissipative materials displaying similar response, such as conventional elastic-plastic materials and ferroelastics. Reverse phase transformation being a dissipative process itself increases the toughness enhancement [12]. However, for increasing temperatures, although the material's tendency to undergo reverse transform increases, so does the critical stress level required for martensitic transformation which has the opposite effect on the toughening response of SMAs. The net outcome is lower levels of fracture toughness enhancement for increasing nominal temperatures, which is essentially what Freed and Banks-Sills [24] reported when they argued that reverse phase transformation can significantly reduce the resistance increase.

The isothermal assumption used in all the aforementioned studies is valid for a range of strain rates within the regime of quasi-static processes even for complex geometries and loadings [25, 26]. At higher strain rates, however, depending on the geometry, convective boundary conditions, and associated heat transfer, the generation or absorption of latent heat may have a strong impact on the deformation response and the stress-strain hysteresis loop area of SMAs as shown experimentally [27-30], and in turn to the fracture behavior of these materials. Given that the energy dissipation during a loading-unloading process is lowest under the extreme case of adiabatic conditions [28], it is expected that the actual transformation toughening in real applications of SMA components would be higher than the predicted value for the adiabatic case. For the NiTi material system investigated in [13], the toughening loss due to thermomechanical coupling for mode I fracture was less than $20 \%$ in the temperature range of interest.

This work focuses on the effect of thermomechanically-induced phase transformation on the driving force for crack growth of a static crack, as a first attempt to understand SMA response during actuation in the presence of a crack. The prototype infinite center-crack problem is analyzed using the finite element method. The thermomechanical loading path used in the analysis is an 
idealization of typical loading paths that utilize SMAs as actuators subject to constant external mechanical loading under temperature variations. The driving force for crack growth is identified to be the crack-tip energy release rate, which is calculated using the Virtual Crack Closure Technique (VCCT).

The remainder of the paper is organized as follows. The constitutive material behavior is described in Section 2. In Section 3, the problem of an infinite center-cracked SMA plate subjected to global thermomechanically-induced phase transformation is formulated in a finite element setting. The obtained results on the crack-tip mechanical fields and driving force for crack growth are given in Section 4. Finally, in Section 5 the key findings are summarized.

\section{Thermomechanical SMA Constitutive Model}

The proposed model relies on the unified model for polycrystalline SMAs proposed by Boyd and Lagoudas [31]. It is developed within the framework of continuum thermodynamics and adopts the classical rate-independent smallstrain flow theory for the evolution equations of the transformation strains.

Within the context of isotropic elastic response, the increments of the strain tensor components, $d \varepsilon_{i j}$, are given as

$$
d \varepsilon_{i j}=S_{i j k l} d \sigma_{k l}+d S_{i j k l} \sigma_{k l}+d \varepsilon_{i j}^{t},
$$

where $\sigma_{i j}, \varepsilon_{i j}^{t}$ are the Cartensian components of the stress tensor and of the transformation strain tensor, respectively, and $S_{i j k l}$ are the components of the 'current' compliance tensor. The thermoelastic strains are an order of magnitude smaller than the transformations strains, and thus neglected. Throughout this paper, standard Einstein notation is used with summation over repeated indices assumed. The 'current' compliance tensor varies with the martensite volume fraction $\xi$ as $S_{i j k l}=(1-\xi) S_{i j k l}^{A}+\xi S_{i j k l}^{M}$, where $S_{i j k l}^{A}$ and $S_{i j k l}^{M}$ are the components of the compliance tensor of austenite and martensite, respectively. The assumption of elastic isotropy for both the austenitic and martensitic phases reads as $S_{i j k l}^{\alpha}=\frac{1+\nu_{\alpha}}{2 E_{\alpha}}\left(\delta_{i l} \delta_{j k}+\delta_{i k} \delta_{j l}\right)-\frac{\nu_{\alpha}}{E_{\alpha}} \delta_{i j} \delta_{k l}$, where the index $\alpha$ stands for A in the case of austenite and for $\mathrm{M}$ in the case of martensite. $E_{\alpha}, \nu_{\alpha}$ denote the Young's modulus and Poisson's ratio of the two phases, respectively, and $\delta_{i j}$ is Kronecker's delta.

An evolution equation of the transformation strain is defined so that it is related to the evolution of martensite volume fraction $\xi$,

$$
d \varepsilon_{i j}^{t}=\Lambda_{i j} d \xi, \quad \Lambda_{i j}=\left\{\begin{array}{l}
\Lambda_{i j}^{f w d}, d \xi>0 \\
\Lambda_{i j}^{r e v}, d \xi<0
\end{array}\right.
$$

where, $\Lambda_{i j}$, the components of the direction tensor, are defined as

$$
\Lambda_{i j}^{f w d}=\frac{3}{2} \frac{H^{c u r}}{\bar{\sigma}} s_{i j}, \quad \Lambda_{i j}^{r e v}=\frac{\varepsilon_{i j}^{t}}{\xi} .
$$


Here, $H^{\text {cur }}$ is the uniaxial transformation strain magnitude for complete transformation, $\bar{\sigma}=\sqrt{\frac{3}{2} s_{i j} s_{i j}}$ is the Mises equivalent stress and $s_{i j}=\sigma_{i j}-\sigma_{k k} \delta_{i j} / 3$ are the stress deviator components. During forward transformation, the transformation strain is oriented by the direction of the deviatoric stress, which motivates the selected $J_{2}$ form of the direction tensor. During reverse phase transformation, it is assumed that the direction and magnitude of the transformation strain recovery is governed by the average orientation of the martensite at transformation reversal (the cessation of forward transformation, be it partial or full). This definition allows to return to a zero transformation strain for every state with a null martensite volume fraction. $H^{\text {cur }}$ is a function of the stress state since most SMA materials do not exhibit a constant maximum attainable transformation strain at all stress levels. A saturated value of maximum attainable transformation strain, $H_{\text {sat }}$, is reached at a high stress level, which is dependent on the SMA material as well as the processing conditions for a polycrystalline material, resulting in different crystallographic and morphological textures, for example. Following this observation, the maximum transformation strain $H^{\text {cur }}$ is represented by the following decaying exponential function

$$
H^{\text {cur }}(\bar{\sigma})=H_{\text {sat }}\left(1-e^{-k \bar{\sigma}}\right),
$$

where the parameter $k$ controls the rate at which $H^{\text {cur }}$ exponentially evolves from 0 to $H_{\text {sat }}$.

During transformation, the stress tensor components should remain on the transformation surface

$$
\Phi=0, \quad \Phi=\left\{\begin{array}{l}
\Phi^{f w d}=\pi^{f w d}-Y_{0}, d \xi>0, \\
\Phi^{r e v}=-\pi^{r e v}-Y_{0}, d \xi<0,
\end{array}\right.
$$

with $\pi^{f w d}, \pi^{r e v}$ being the thermodynamic driving forces for forward and reverse transformation, respectively, and $Y_{0}$ is the critical value of the thermodynamic force to both initiate and sustain forward and reverse phase transformation. The thermodynamic driving force for forward transformation is written as

$$
\pi^{f w d}=\sigma_{i j} \Lambda_{i j}^{f w d}+\frac{1}{2} \Delta S_{i j k l} \sigma_{i j} \sigma_{k l}+\rho \Delta s_{0} T-\rho \Delta u_{0}-f^{f w d},
$$

where

$$
f^{f w d}=\frac{1}{2} \alpha_{1}\left[1+\xi^{n_{1}}-(1-\xi)^{n_{2}}\right]+\alpha_{3},
$$

and for reverse transformation

$$
\pi^{r e v}=\sigma_{i j} \Lambda_{i j}^{r e v}+\frac{1}{2} \Delta S_{i j k l} \sigma_{i j} \sigma_{k l}+\rho \Delta s_{0} T-\rho \Delta u_{0}-f^{r e v},
$$

where

$$
f^{r e v}=\frac{1}{2} \alpha_{2}\left[1+\xi^{n_{3}}-(1-\xi)^{n_{4}}\right]-\alpha_{3}
$$




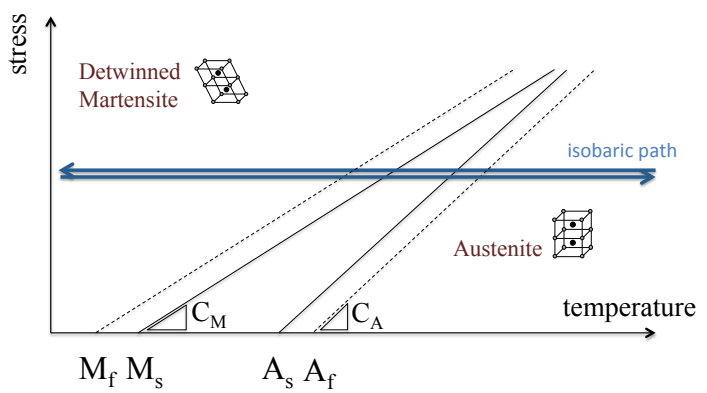

Figure 2: Stress-temperature phase diagram. A thermal cycle at constant applied load.

$f^{f w d}$ and $f^{r e v}$ are functions describing the transformation hardening behavior during forward and reverse phase transformation, respectively. $s_{0}$ and $u_{0}$ are the specific entropy and internal energy, respectively, $\rho$ is the density, $\Delta$ denotes the difference in property between the martensitic and the austenitic states, $\alpha_{i}$ $(i=1,2,3)$ and $n_{i}(i=1,2,3,4)$ are coefficients that assume real number values.

Given these constitutive relations the following model parameters must be calibrated: (i) the elastic parameters of martensite and austenite, (ii) parameters contained in the functional form of the maximum transformation strain $H^{\text {cur }}(\bar{\sigma})$, and (iii) six model parameters $\left(\rho \Delta s_{0}, \rho \Delta u_{0}, \alpha_{1}, \alpha_{2}, \alpha_{3}, Y_{0}\right)$ that are characteristic of the martensitic transformation. The common material properties that are used to calibrate the model are $E_{A}, E_{M}, \nu_{A}, \nu_{M}, H_{s a t}, M_{s}, M_{f}, A_{s}$, $A_{f}, C_{M}$, and $C_{A} . M_{s}, M_{f}, A_{s}$ and $A_{f}$ are the martensitic-start, martensiticfinish, austenitic-start and austenitic-finish temperatures at zero load, respectively, and $C_{M}$ and $C_{A}$ are the forward and reverse transformation slopes in the stress-temperature phase diagram, respectively (Figure 2). The elastic constants can be calculated directly from isothermal stress-strain curves where loads are applied at temperatures outside the transformation regions. The parameters for $H^{\text {cur }}(\bar{\sigma})$ can be calibrated directly from isobaric material testing, where the value of $k$ in particular is chosen to best fit the experimental trend. The remaining six parameters are calibrated by considering the conditions under which forward transformation begins and ends in the stress-temperature space [32]. The exponents $n_{i}(i=1,2,3,4)$ do not have an associated material property but are directly chosen to best fit the two corners of the forward transformation plots.

\section{Problem Formulation and Method of Solution}

The effect of thermomechanically-induced phase transformation on the driving force for crack growth in SMAs is analyzed via the finite element method. The analysis is performed on an infinite SMA plate containing a central crack of length $2 a$, subjected to in-plane uniform uniaxial tensile stress at infinity, $\sigma_{\infty}$, 
in the direction perpendicular to the crack axis under temperature variations and plane strain conditions (Figure 3). Due to the mode I symmetry of the problem only the upper quarter plane is analyzed. A system of co-ordinates $\left(x_{1}, x_{2}\right)$ is taken such as the origin lies at the center of the crack and the $x_{1}$-axis is extending along the line of the crack. A finite element mesh of eight-noded, isoparametric quadrilaterals elements is designed for the analysis (Figure 4).

The mechanical load is applied at a constant ambient temperature, $T_{h}$, which is assumed greater than the austenitic-finish temperature, $A_{f}$, and lower than $M_{d}$, so that phase transformation will take place for sufficient load levels. The resulting uniaxial tensile stress at infinity, $\sigma_{\infty}$, is assumed sufficiently smaller than the stress required for initiation of martensitic transformation, $\sigma^{M_{s}}$, at the given temperature $T_{h}$, so that small scale transformation conditions prevail, according to which the size of the transformation zone is small compared to the crack length $2 a$. Moving from the boundary inwards to the crack tip, a region of partially transformed material will be first encountered and closer to the crack tip a region of fully transformed material. Then the temperature is cycled between $T_{h}$ and a temperature $T_{c}$, which is lower than the martensiticfinish temperature, $M_{f}$, to ensure that the entire material is transformed from austenite to martensite at the end of cooling. The temperature distribution is assumed uniform throughout the material at every instant, i.e., latent heat effects are neglected by assuming the rate of both the mechanical and thermal loading being sufficiently slow with respect to the time rate of heat transfer by conduction. In real applications of SMA components, the generation of entropy and heat during phase transformation may violate this assumption, influencing the phase transition stress-values that are temperature dependent and in turn the fracture response of SMAs due to the resulting strong thermomechanical coupling. The effect of latent heat on the driving force for crack growth during thermal actuation of SMAs will be examined in a subsequent publication.

The incremental response of the material inside the fully transformed zone surrounding the crack tip at all times is linear elastic, and the fields are characterized by an unknown crack-tip energy release rate, $G_{I}$. The VCCT is employed in the analysis for computing $G_{I}[33-35]$. VCCT is an extension of the classical crack closure method based on the Irwin's crack closure integral [36]. In the case of the two-dimensional eight-noded elements placed in the crack front, the method yields

$$
G_{I}=-\frac{1}{2 \Delta a}\left[F_{2}^{i}\left(u_{2}^{l}-u_{2}^{l^{*}}\right)+F_{2}^{j}\left(u_{2}^{m}-u_{2}^{m^{*}}\right)\right],
$$

where $F_{2}^{i}$ and $F_{2}^{j}$ indicate the perpendicular to the crack plane nodal forces at the tip and at the mid-side node in front of the crack, respectively (Figure 5). Also, $u_{2}^{l}, u_{2}^{l^{*}}$ and $u_{2}^{m}, u_{2}^{m^{*}}$ represent the opening displacement of the upper and lower crack surfaces at the four nodes placed behind the crack tip [34]. The calculated $G_{I^{-}}$-value approximates the crack-tip energy release rate on the basis of the following assumptions: (i) a crack extension of $\Delta a$ from $a+\Delta a$ (node i) to $a+2 \Delta a$ (node $k$ ) does not significantly alter the state at the crack tip, and (ii) the energy released when the crack is extended by $\Delta a$ from $a+\Delta a$ to 


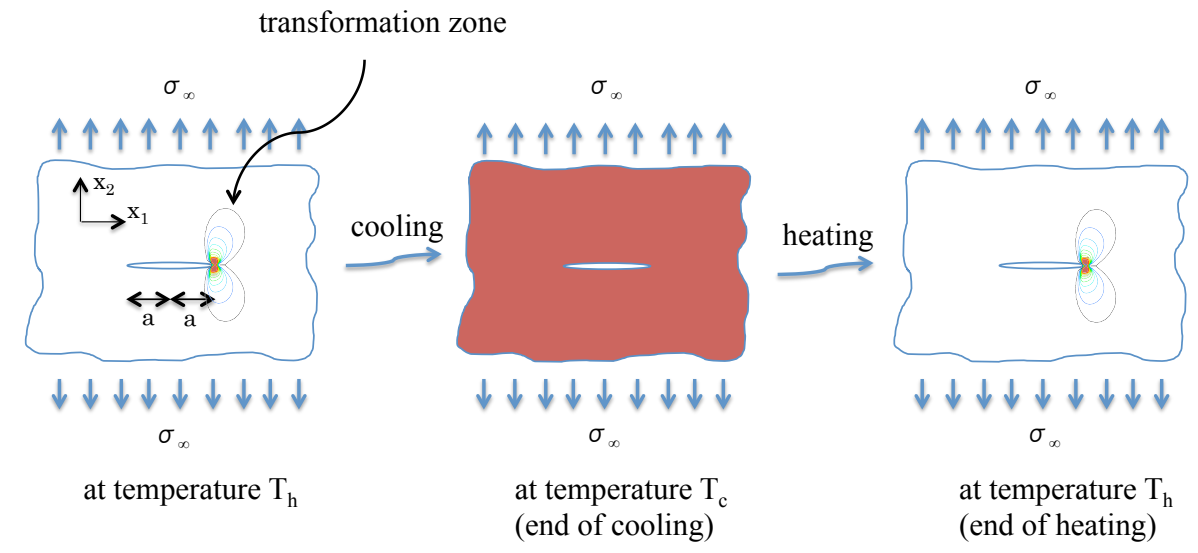

Figure 3: Boundary value problem for an infinite center-cracked SMA plate subjected to a thermal cycle under constant applied load. The region of fully transformed material is represented with the red color.

$a+2 \Delta a$ is equal to the energy required to close the crack between location $i$ and $k$ (Figure 5 ).

The two aforementioned assumptions are considered acceptable for the constitutive material behavior used in the analysis, which results in a linear elasticallydeformed martensite region surrounding the crack tip. Specifically, it is assumed that during virtual crack growth, under which the temperature and tractions at all boundary points are kept constant, most of the dissipation within the cracked body comes from the separation work, i.e., the contribution of the rate of the transformation strain work with respect to crack length, $\partial U^{t} / \partial a$, in the energy balance equation [37]

$$
\frac{\partial W}{\partial a}-\frac{\partial U^{e}}{\partial a}=C+\frac{\partial U^{t}}{\partial a},
$$

is small compared to the separation work $C$ since the region close to the crack tip is fully transformed. In the above equation, $\partial W / \partial a$ is the work rate supplied by the external loads and $\partial U^{e} / \partial a$ is the rate of elastic strain energy. Essentially it is assumed that at constant temperature and tractions at the external boundary a differential crack growth results mainly in a stress redistribution in the region of elastically-deformed martensite close to the crack tip. Further justifications for the VCCT in evaluating the crack-tip energy release rate are offered by the calculated stress fields close to the crack tip and the crack-tip $J$-value in Section 4 below.

Within this model for fracture of SMAs, dimensional analysis and manipulation of the constitutive law dictate that the normalized stresses, $\sigma_{i j} / \sigma_{\infty}$, strains, $\varepsilon_{i j} / H_{s a t}$, and temperature, $C_{M}\left(T-M_{s}\right) / \sigma_{\infty}$, will be dependent on the 
$\sigma_{\infty}$

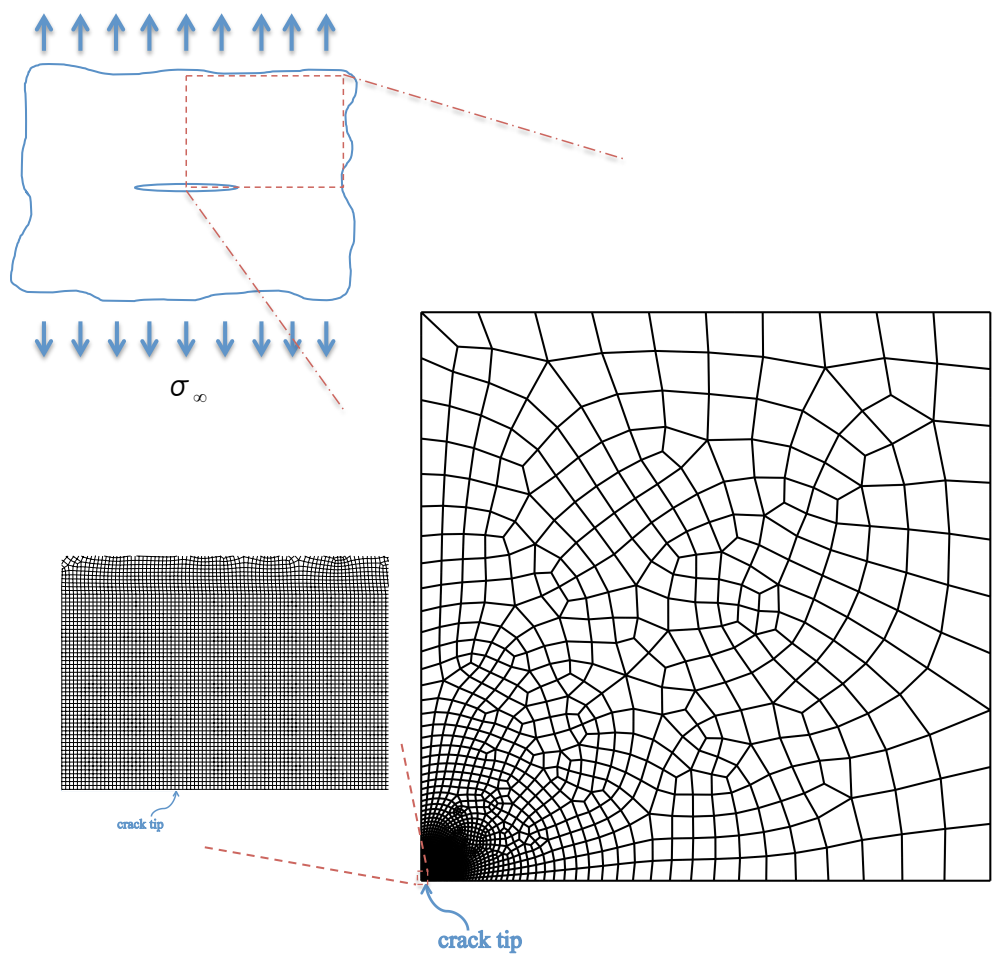

Figure 4: The finite element mesh used in the calculations. The mesh comprises 10078 quadrilateral elements.

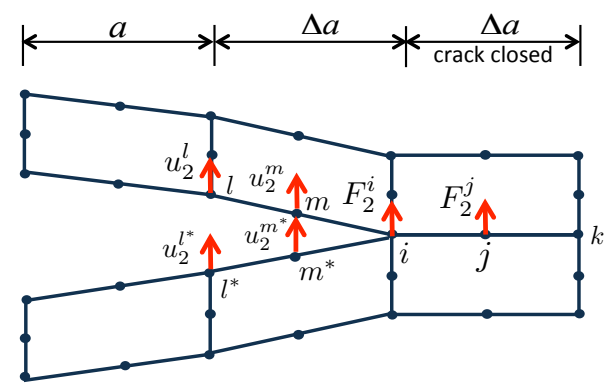

Figure 5: VCCT for eight-noded elements. 
following dimensionless parameters

$$
\begin{gathered}
\frac{E_{A} H_{s a t}}{\sigma_{\infty}}, k \sigma_{\infty}, \frac{C_{M}\left(A_{s}-M_{s}\right)}{\sigma_{\infty}}, \frac{C_{M}\left(A_{f}-A_{s}\right)}{\sigma_{\infty}}, \frac{C_{M}\left(M_{s}-M_{f}\right)}{\sigma_{\infty}}, \frac{C_{M}}{C_{A}}, \\
\frac{E_{M}}{E_{A}}, \nu_{M}, \frac{\nu_{M}}{\nu_{A}},
\end{gathered}
$$

which, under the convention of tensile stresses being positive, are subject to the following inequalities

$$
\frac{C_{M}\left(A_{f}-A_{s}\right)}{\sigma_{\infty}} \geq 0 \text { and } \frac{C_{M}\left(M_{s}-M_{f}\right)}{\sigma_{\infty}} \geq 0,
$$

since $M_{f} \leq M_{s}, A_{s} \leq A_{f}$. Note that, in what follows, the Poisson's ratios of the two phases are assumed equal, i.e., $\nu_{A}=\nu_{M}=\nu$, which is the case for most SMA material systems.

\section{Results}

\subsection{Stress field close to the crack tip}

Some features of the crack-tip mechanical fields are now presented. Contours of martensite volume fraction during thermomechanical loading are presented in Figure 6. The size of the stress-induced martensitic zone resulting from the applied mechanical load prior to cooling is approximately 50 times smaller than the crack length, in accordance with the small scale transformation assumption adapted for the calculations. During cooling, transformation occurs first in a fan in front of the crack tip and expands to regions behind the crack tip as the temperature decreases, while during heating, reverse phase transformation occurs first behind the crack tip, where the stress levels are lower, and in regions in front of the crack tip at higher values of temperature. The thermomechanicallyinduced phase transformation results in a stress redistribution that changes the crack-tip stress field, as shown in Figure 7, in which the von Mises stress and mean normal stress, $\sigma_{m}=1 / 3 \cdot\left(\sigma_{11}+\sigma_{22}+\sigma_{33}\right)$, distributions at the crack tip are presented prior to thermal cycling (i.e., at $T=T_{h}$ ) and at the end of cooling (i.e., at $T=T_{c}$ ).

The numerical results suggest that close to the crack tip the stresses have a $1 / \sqrt{r}$ radial asymptotic behavior during thermal cycling. In Figure 8 , the angular dependence of the SMA stress field close to the crack tip at the end of cooling is compared to the angular dependence of the calculated stress field for an isotropic linear elastic solid. The values of the non-dimensional parameters used in the calculations are those of Figure 9. According to the calculations, the stress field close to the crack tip for an SMA material is equivalent to that of an isotropic linear elastic material. Therefore, the stress and strain fields close to the crack tip can be characterized by either $G_{I}$ or the crack-tip stress intensity factor, $K_{I}=\sqrt{E_{M} G_{I} /\left(1-\nu^{2}\right)}$ (see the vertical axis label in Figure 8). This result, apart from justifying the assumption of a single parameter being capable 
of describing the mechanical fields close to the crack tip, validates the VCCT in calculating that parameter. However, it only holds true for sufficient large sizes of the fully transformed martensitic region close to the crack tip. At higher temperatures close to $T_{h}$ and small sizes of the fully transformed martensitic region, which dictate an even smaller size for the region of $K_{I}$-dominance, a discrepancy between the calculated SMA stress field and the mode I elastic asymptotic $K_{I}$-field is observed. It is likely that the stress fields in those cases are evaluated outside the $K_{I}$-dominance region, where higher order terms in the series expansion of the crack-tip stress field are significant [12].

\subsection{Energy release rate during a thermal cycle at constant applied load}

The effect of thermomechanically-induced phase transformation on the driving force for crack growth during a thermal cycle at constant applied load, as given by the ratio $G_{I} / G_{\infty}$, is examined next, where $G_{\infty}$ corresponds to the value of the energy release rate due to the mechanical load alone, i.e., at $T=T_{h}$ prior to thermal cycling. As shown in Figure 9, during cooling, the energy release rate initially increases until it reaches a peak and then decreases before attaining a value at temperature $T=M_{f}$, at which the entire material is fully transformed, which remains constant under cooling at lower temperatures. During heating, the energy release rate starts increasing at temperature $T=A_{s}$, at which reverse phase transformation initiates, then, after reaching a peak, it decreases before attaining, eventually, a constant value. Thus, if crack growth is assumed to occur when the crack-tip energy release rate reaches a material specific critical value related to the martensitic phase, then crack growth may occur during thermal variations under constant applied loading as a result of phase transformation. It should be emphasized that since the thermal cycling involves temperatures that are lower than $M_{d}$ and in the presence of a martensitic zone close to the crack tip throughout the thermal cycle, the austenite specific critical value of the driving force for crack growth that may be different than that of martensite is not of relevance in the calculations.

Note that (i) the far-field (i.e., at infinity) material starts transforming into martensite during cooling at temperature $T=M_{s}+\sigma_{\infty} / C_{M}$, at which the farfield stress, $\sigma_{\infty}$, is equal to the stress required for initiation of phase transformation $\sigma^{M_{s}}=C_{M}\left(T-M_{s}\right)$ (for the material properties chosen in Figure 9, this temperature happens to coincide with $\left.C_{M}\left(A_{s}-M_{s}\right) / \sigma_{\infty}\right)$. At that temperature, which corresponds to "global"- scale transformation, the increase of the energy release rate is about $30 \%\left(G_{I} / G_{\infty} \simeq 0.3\right)$. (ii) The peak $G_{I} / G_{\infty}$-values during the thermal cycle, which are approximately equal to 6.5 , are not attributed just to the elastic moduli mismatch. The mismatch between the Young's moduli yields wide variations of the stress fields due to the material constraint in planestrain conditions, however, numerical calculations for $E_{M}=E_{A}$ still show a similar substantial enhancement of the energy release rate (see Figure 17) that should be attributed to stress redistribution caused by large scale transformation, and (iii) the normalized ratio $G_{I} / G_{\infty}$ is independent of the crack length $2 a$, an expected result since the crack length is the only length scale in the considered fracture problem as the plate is infinite. 


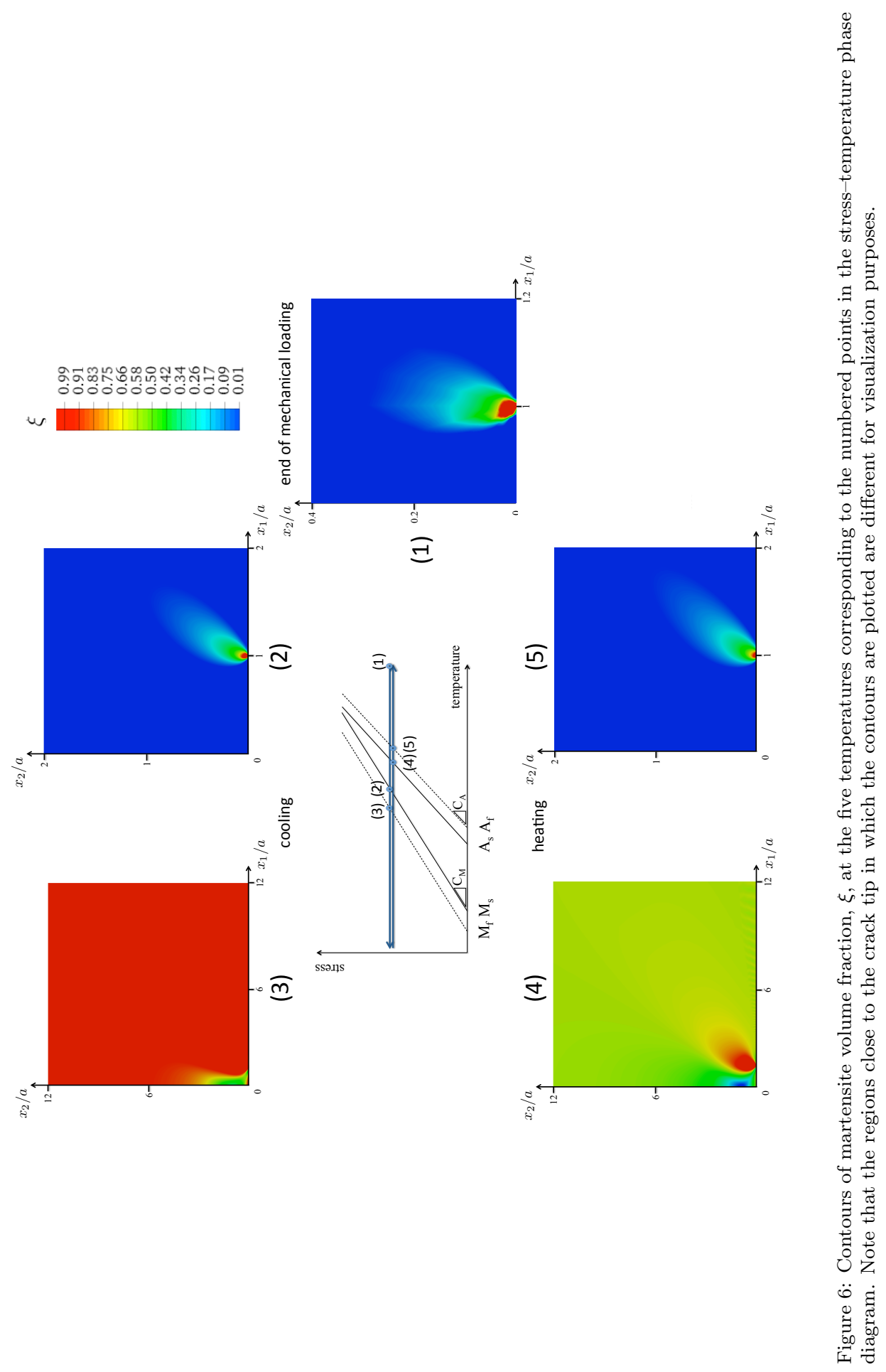




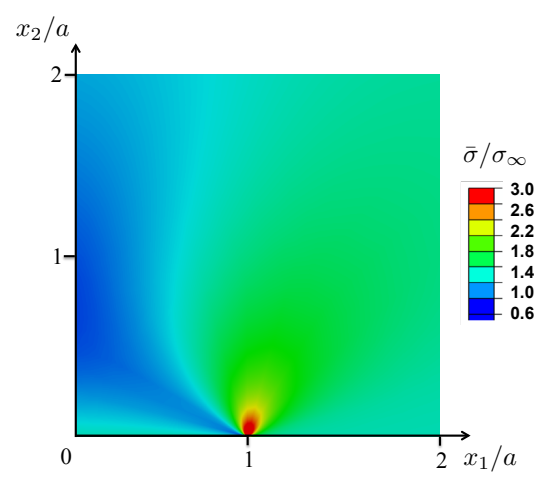

(a) von Mises stress at $T=T_{h}$, prior to thermal cycling.

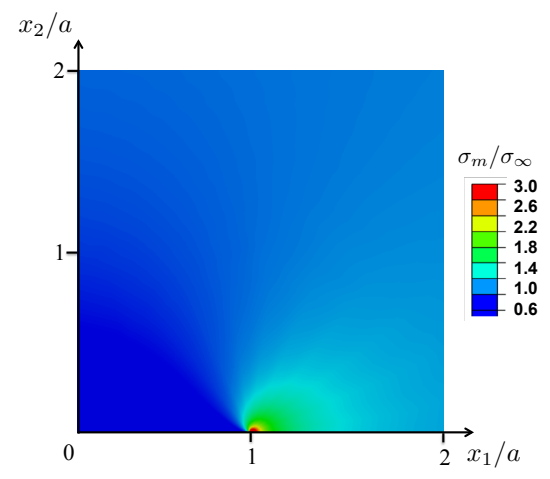

(c) Mean normal stress at $T=T_{h}$, prior to thermal cycling.

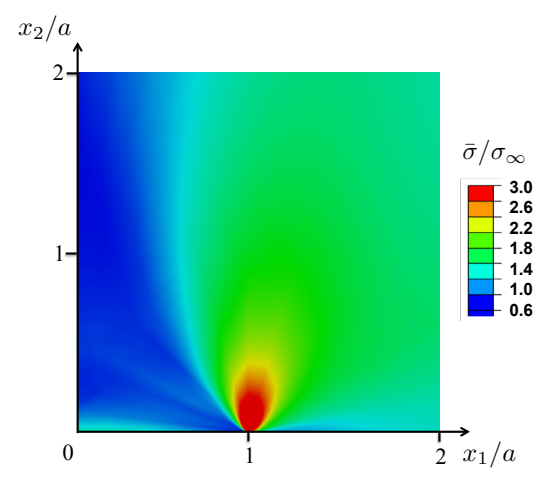

(b) von Mises stress at the end of cooling.

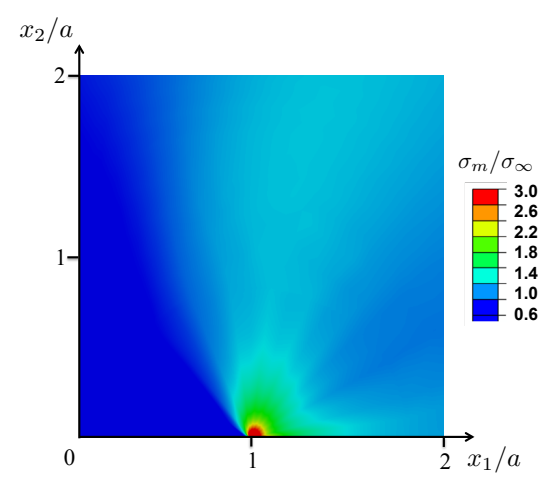

(d) Mean normal stress at the end of cooling.

Figure 7: Normalized von Mises stress, $\bar{\sigma} / \sigma_{\infty}$, and mean normal stress, $\sigma_{m} / \sigma_{\infty}$, distributions near the crack tip. 


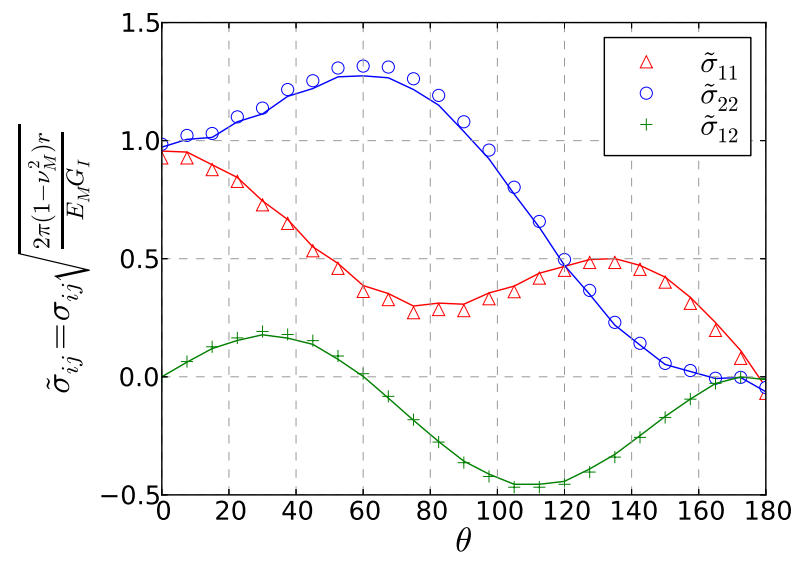

Figure 8: Angular distribution of stresses close to the crack tip at the end of cooling. The markers are the numerical results for the SMA material and the solid lines are numerical results for an elastic material with the properties of martensite. The $1 / \sqrt{r}$ radial dependence has been accounted for within the normalization.

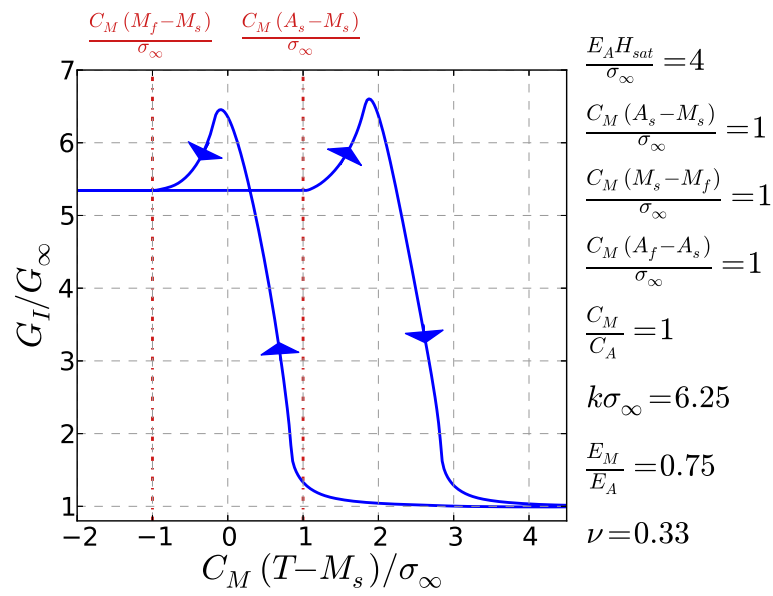

Figure 9: Normalized energy release rate, $G_{I} / G_{\infty}$, versus normalized temperature, $C_{M}(T-$ $\left.M_{s}\right) / \sigma_{\infty}$. 
4.3. An insight into the stress redistribution caused from large scale transformation

An insight into the presented results concerning the effect of actuation on the crack-tip energy release rate $G_{I}$, or equivalently on the stress intensity factor $K_{I}$ in SMAs, can be obtained from the enhancement/reduction in the stress intensity factor, $d K_{I}$, due to two differential elements of area $d A$ undergoing a transformation strain. These two are assumed symmetrically located with respect to the plane of the crack so that mode I conditions prevail. The first is located at $(r, \beta)$ with respect to the crack tip and is characterized by the stress-free strains $\left\{\varepsilon_{11}^{t}, \varepsilon_{22}^{t}, \varepsilon_{12}^{t}\right\}$ for plane strain loading, whereas the second is located at $(r,-\beta)$ and undergoes the stress-free strains $\left\{\varepsilon_{11}^{t}, \varepsilon_{22}^{t},-\varepsilon_{12}^{t}\right\}$. For differential elements close to the tip, $d K_{I}$ can be approximated by the closed form expression obtained for a semi-infinite crack in an infinite domain $[38,39]$,

$$
d K_{I}=\frac{1}{\sqrt{8 \pi}} \frac{E d A}{1-\nu^{2}} r^{-3 / 2} M\left(\varepsilon_{\gamma \delta}^{t}, \beta\right)
$$

where

$$
M\left(\varepsilon_{\gamma \delta}^{t}, \beta\right)=\varepsilon_{\alpha \alpha}^{t} \cos \frac{3 \beta}{2}+3 \varepsilon_{12}^{t} \cos \frac{5 \beta}{2} \sin \beta+\frac{3}{2}\left(\varepsilon_{22}^{t}-\varepsilon_{11}^{t}\right) \sin \frac{5 \beta}{2} \sin \beta,
$$

with the Greek indices ranging over 1 to 2 and the repeated Greek index indicating a sum over 1 and 2. For differential elements farther afield the value of $d K_{I}$ may differ significantly from the above expression. From (13) and (14) it can be concluded that there is fan ahead of the crack tip, such that any transformed material which falls within this fan and at sufficiently small distances from the crack tip increases the near-tip intensity and consequently the energy release rate, while transformed material behind this fan reduces that intensity. For pure dilatational transformation, the fan is of $120^{\circ}$.

On the basis of the above analysis, although it is quantitatively valid only at sufficiently small distances from the crack tip, and by considering that for the specific crack configuration considered here intense shearing is confined in planes emanating at $45^{\circ}$ from the tensile direction, it is plausible that the increase of crack-tip energy release rate during cooling is due to global-scale transformation occurring mostly in regions in front of the crack tip. The subsequent decrease once the peak is reached should then be attributed to transformation occurring in regions behind the crack tip (Figure 10). A similar argument can explain the behavior of the crack-tip energy release rate during heating. The material first reverse transforms in regions behind the crack tip where the stresses are low resulting in an increase of the energy release rate since the shielding effect of the transformation strains in such regions is lost. Then the material reverse transforms in regions in front of the crack tip where the transformation strains have an anti-shielding effect resulting in a decrease of the energy release rate.

Expression (13) is readily valid for small scale transformation conditions and yields, if integrated on the classical "butterfly" transformation domain in plane strain loading, a crack-tip energy release rate that can be approximated 


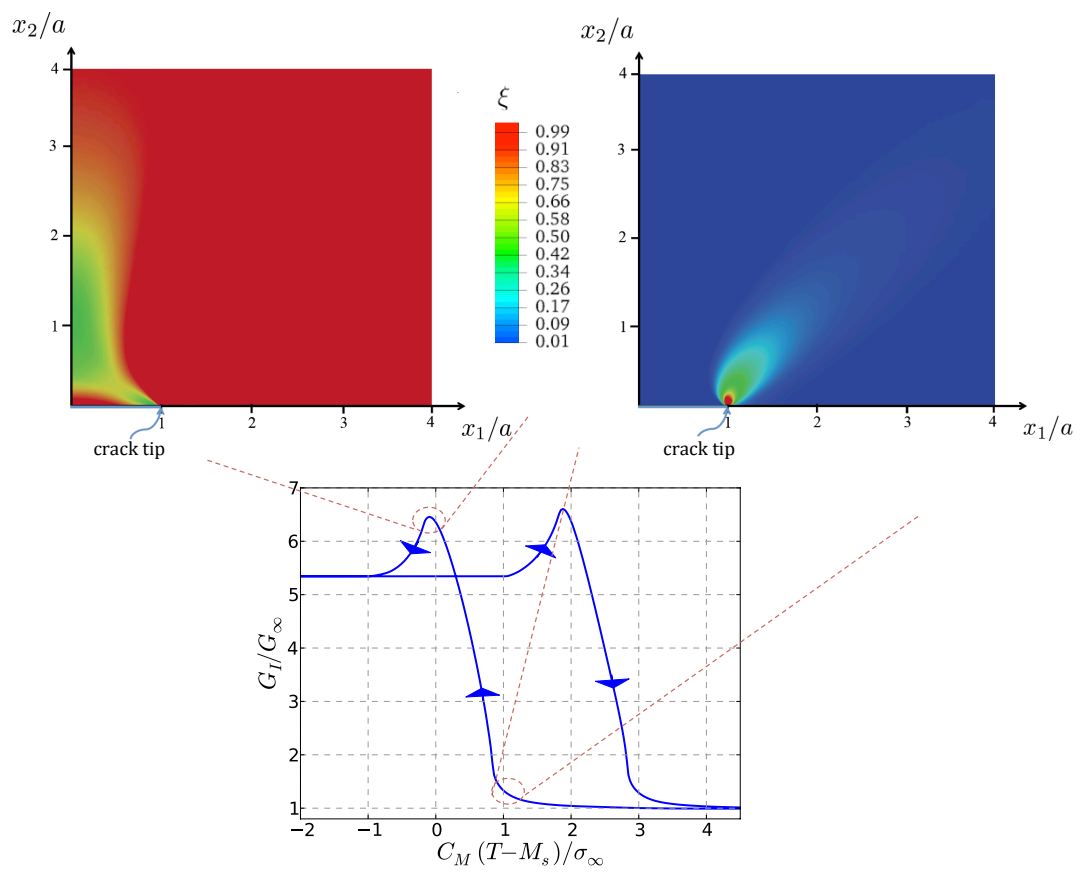

Figure 10: Martensite volume fraction, $\xi$, during cooling. The martensite volume fraction distribution during heating is similar to the one during cooling for the same values of $G_{I} / G_{\infty}$. 
by the far-field energy release rate [39]. This can be observed in Figure 9 for temperature values higher than $T=M_{s}+\sigma_{\infty} / C_{M}$, i.e., at normalized temperature values $C_{M}\left(T-M_{s}\right) / \sigma_{\infty}$ higher than 1. At temperatures lower than $M_{s}+\sigma_{\infty} / C_{M}$, where a substantial increase of the energy release rate is observed during cooling, the far-field material is transforming into martensite and global-scale transformation conditions prevail. The transformation zone size and shape at such temperatures are determined by the dimensions and shape of the crack configuration. The observed substantial enhancement of the energy release rate should therefore be attributed to the stress redistribution caused by global-scale transformation while the levels of enhancement are tied solely to actuation of a center-cracked SMA plate subjected to constant in-plane loading.

\subsection{Non-proportionality of loading}

The non-proportionality of loading is investigated via $J$-integral calculations [40-42]. A strong path-dependency of the $J$-integral is expected similarly to center-cracked panels of conventional elastic-plastic materials loaded in tension under large scale yielding [43]. Since the path-independence of $J$ rigorously holds for proportional loading conditions holding everywhere in the domain, it's path-dependency would indicate non-proportional loading.

Singular crack tip elements are used for the $J$-integral evaluation, shaped as isosceles triangles focused into the crack tip and placed in angular intervals of $\pi / 24$. These elements are created by collapsing one side of the eight-noded quadrilaterals and assigning each of the crack tip nodes common degrees of freedom, with the midside nodes on the sides connected to the crack tip moved on the $1 / 4$ points nearest the crack tip. The interpolation function for such elements exhibits a singularity of the form $\mathrm{Br}^{-1 / 2}$ in displacement derivatives.

The values of the $J$-integral

$$
J=-\int_{\Gamma}\left[W n_{1}-\sigma_{j i} n_{j} \frac{\partial u_{i}}{\partial x_{1}}\right] d s,
$$

are computed along circular counterclockwise arcs $\Gamma$ of different radii $R$, centered at the crack tip. $n_{i}$ are the Cartesian components of the outer unit normal to $\Gamma$ and $W=\int \sigma_{i j} d \varepsilon_{i j}$ is the integrated stress work density at a material point. The domain integral method, described in [44-46], is used in the computations

$$
J_{A}=-\int_{A}\left[W \frac{\partial q}{\partial x_{1}}-\sigma_{j i} \frac{\partial q}{\partial x_{j}} \frac{\partial u_{i}}{\partial x_{1}}\right] d A
$$

where $A$ is the region enclosed by an outer circular contour, the first ring of elements directly connected to the crack tip and the crack faces, and $q$ is a function that is equal to unity at the inner boundary of $A$ and equal to zero at the outer boundary. Carka and Landis [47] showed that $J_{A}$ is equal to the radial average of $J$ for all circular paths in $A$.

The $J$-integral is found to be path-dependent as shown in Figure 11. The path-dependency of $J$-integral extends well beyond the region of intense strain 


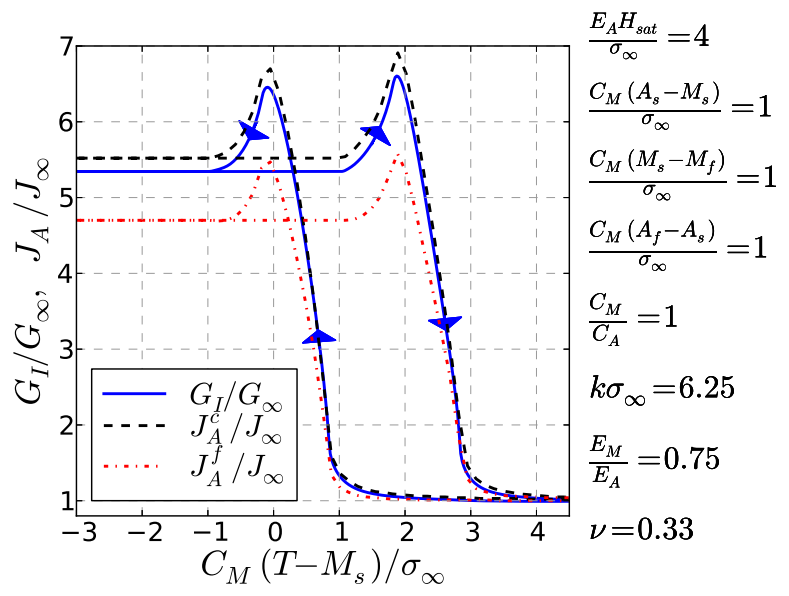

Figure 11: Normalized energy release rate, $G_{I} / G_{\infty}$, and normalized $J$-values, $J_{A} / J_{\infty}$, versus normalized temperature, $C_{M}\left(T-M_{s}\right) / \sigma_{\infty}$. $J_{A}^{c}$ stands for the $J_{A}$-value at the first ring of elements surrounding the crack tip and $J_{A}^{f}$ stands for the $J_{A}$-value at a ring of elements further away from the crack tip.

close to the crack tip. However, the crack-tip $J$-value is very close to the obtained energy release rate value. These two values should in theory be equal. Therefore, the close values obtained using the two different methodologies, i.e., VCCT and the domain integral method, constitute further validation of the VCCT employed in calculating the crack-tip energy release rate.

It should also be noted that the obtained energy release rate values due to cooling at constant load can be approximated by following a different thermomechanical loading path, presented schematically in Figure 12. If instead of first applying the mechanical load, $\sigma_{\infty}$, and then cooling down the material to a temperature $T$, similar enhancement in the energy release rate can be obtained by first cooling down the material to the specific temperature, $T$, and then applying the mechanical load $\sigma_{\infty}$ (Figure 12). The $G_{I}$-values obtained by following the two paths are very close even if global-scale transformation conditions prevail, in spite of the non-proportionality of loading associated with these conditions, as long as the mechanical load in path \#2 is applied at a temperature at which the material is in the austenite phase (Figure 13). Otherwise the two thermomechanical loading paths may yield quite different $G_{I^{-} \text {-values due }}$ to the inherent differences between transformation of austenite into detwinned martensite and detwinning of twinned martensite.

\subsection{Influence of phase transformation metrics on the energy release rate during a thermal cycle at a constant applied load}

The sensitivity of the energy release rate during actuation on the key nondimensional parameters given in Eqn. (12) is examined next. The results to be 


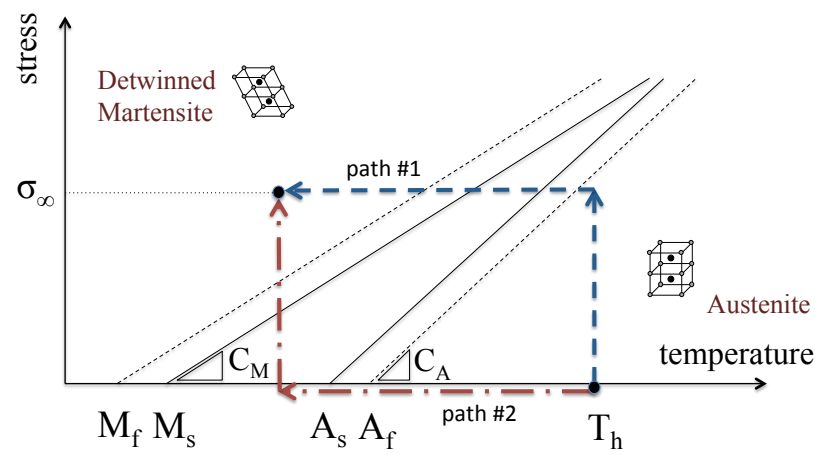

Figure 12: Different thermomechanical-loading paths resulting in approximately equal, within $5 \%$, energy release rates.

presented can be rationalized on the basis of the analysis presented above.

In Figure 14, it can be seen that through out the thermal cycling process the energy release rate increases monotonically as the relative maximum transformation strain, $E_{A} H_{s a t} / \sigma_{\infty}$, increases. Larger relative maximum transformation strains imply higher stress-free strains, which, in accordance to the analysis described above, imply a higher energy release rate. The effect of $k \sigma_{\infty}$ on the energy release rate is similar to that of $E_{A} H_{s a t} / \sigma_{\infty}$, since $k \sigma_{\infty}$ is related to the transformation strain attained at a level of applied mechanical load, which is greater for higher values of $k \sigma_{\infty}$. The effect of $C_{M}\left(A_{s}-M_{s}\right) / \sigma_{\infty}$ on the energy release rate is presented in Figure 15. Higher values of $C_{M}\left(A_{s}-M_{s}\right) / \sigma_{\infty}$ shift reverse phase transformation into a higher temperature range with an analogous effect on the energy release rate vs temperature response. The non-dimensional parameter $C_{M}\left(M_{s}-M_{f}\right) / \sigma_{\infty}$ has an impact (i) on the slope of the energy release rate vs temperature curve during cooling, (ii) the constant value of energy release rate attained once the whole material is fully transformed from austenite to martensite, and (iii) the peak values attained during cooling and heating, all of them getting lower for higher values of $C_{M}\left(M_{s}-M_{f}\right) / \sigma_{\infty}$, i.e., for increasing levels of transformation hardening (Figure 16). Higher values of $C_{M}\left(A_{f}-A_{s}\right) / \sigma_{\infty}$ result in lower slopes of the energy release rate vs temperature curve and peak values during heating. Finally, decreasing values of $E_{M} / E_{A}$ result in higher energy release rates through out the thermal cycling process (Figure 17). From the above sensitivity analysis it can be concluded that the ratio $G_{I} / G_{\infty}$ during thermomechanically-induced phase transformation is lower for material systems with smaller maximum transformation strain, higher martensitic transformation hardening, and smaller Young's moduli mismatch - assuming that the Young modulus of austenite is greater than that of martensite, which is the case for most SMA material systems.

Since most of the non-dimensional parameters listed in Eq. 12 depend on the in-plane uniform uniaxial tensile stress at infinity $\sigma_{\infty}$ and therefore are not 


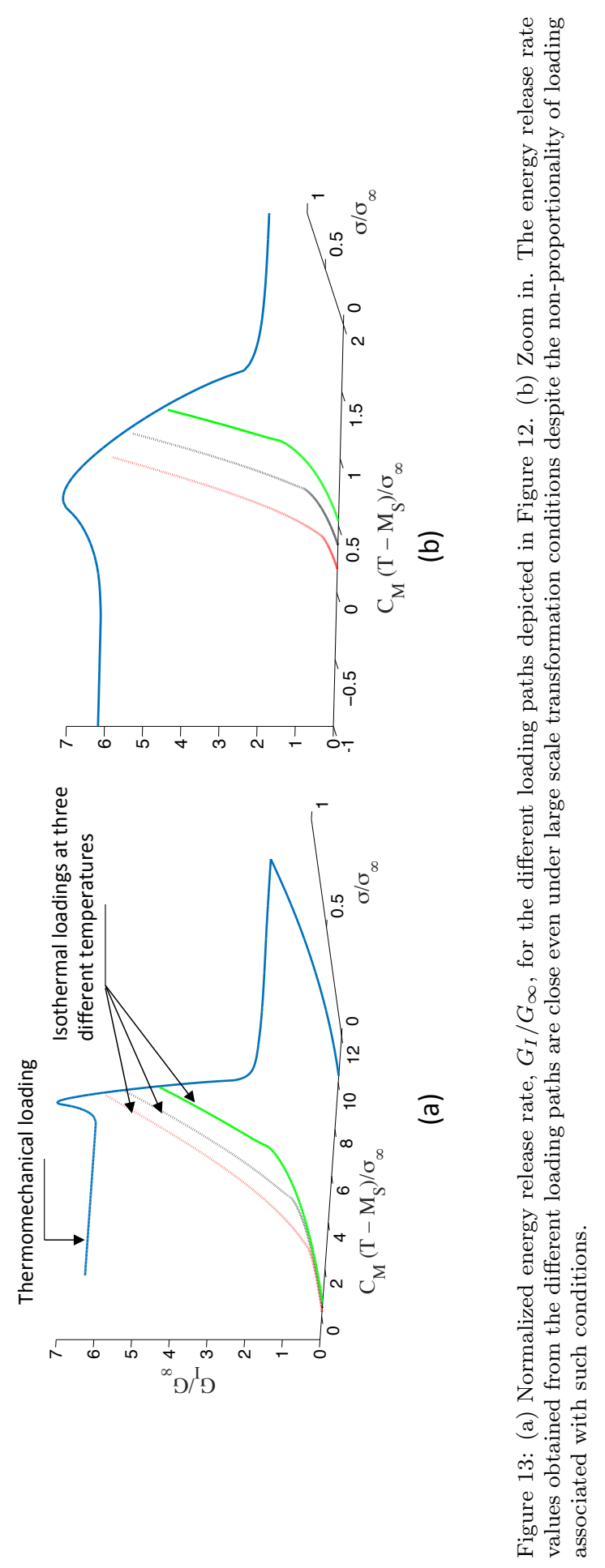




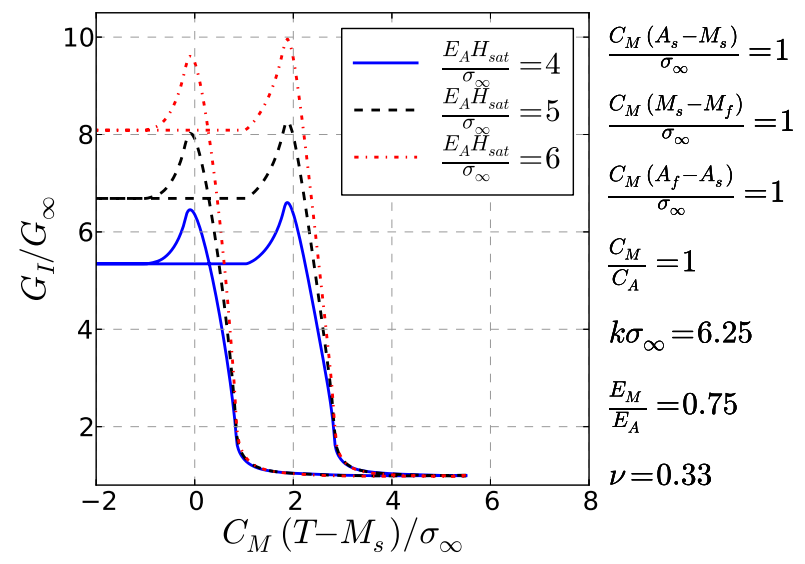

Figure 14: Normalized energy release rate, $G_{I} / G_{\infty}$, versus normalized temperature, $C_{M}(T-$ $\left.M_{s}\right) / \sigma_{\infty}$, for a range of the relative maximum transformation strain, $E_{A} H_{s a t} / \sigma_{\infty}$.

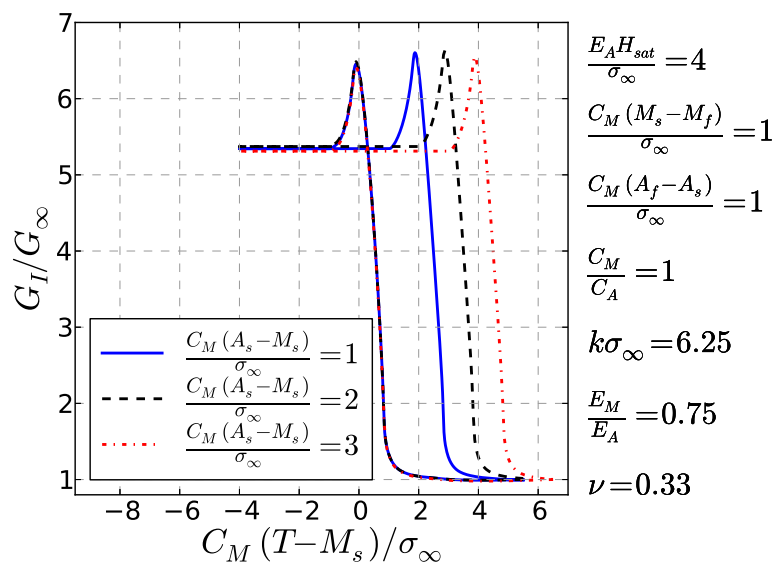

Figure 15: Normalized energy release rate, $G_{I} / G_{\infty}$, versus normalized temperature, $C_{M}(T-$ $\left.M_{s}\right) / \sigma_{\infty}$, for a range of the non-dimensional parameter $C_{M}\left(A_{s}-M_{s}\right) / \sigma_{\infty}$, which is related to the width of the temperature hysteresis. 


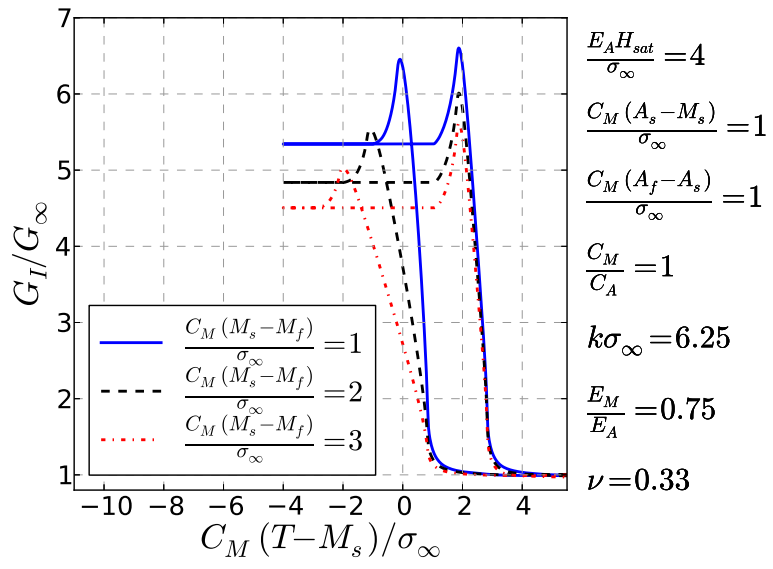

Figure 16: Normalized energy release rate, $G_{I} / G_{\infty}$, versus normalized temperature, $C_{M}(T-$ $\left.M_{s}\right) / \sigma_{\infty}$, for a range of the non-dimensional parameter $C_{M}\left(M_{s}-M_{f}\right) / \sigma_{\infty}$, which is related to the slope of the strain-temperature curve during forward transformation.

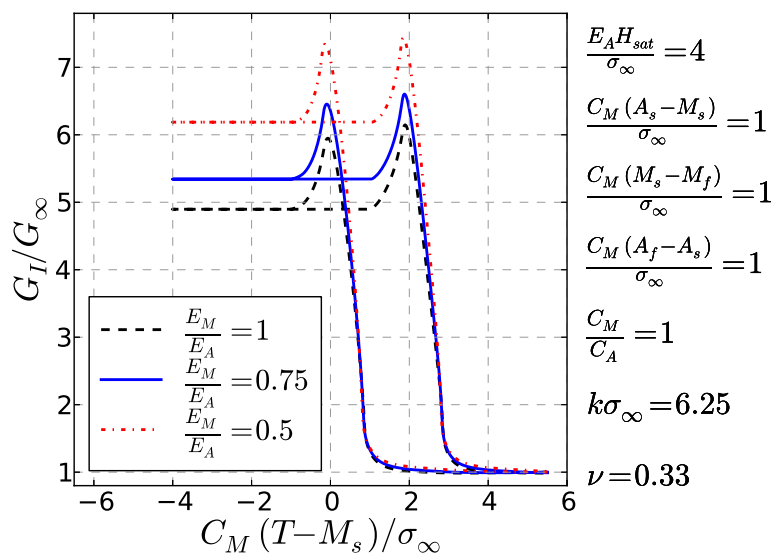

Figure 17: Normalized energy release rate, $G_{I} / G_{\infty}$, versus normalized temperature, $C_{M}(T-$ $\left.M_{s}\right) / \sigma_{\infty}$, for a range of the the relative stiffness, $E_{M} / E_{A}$. 


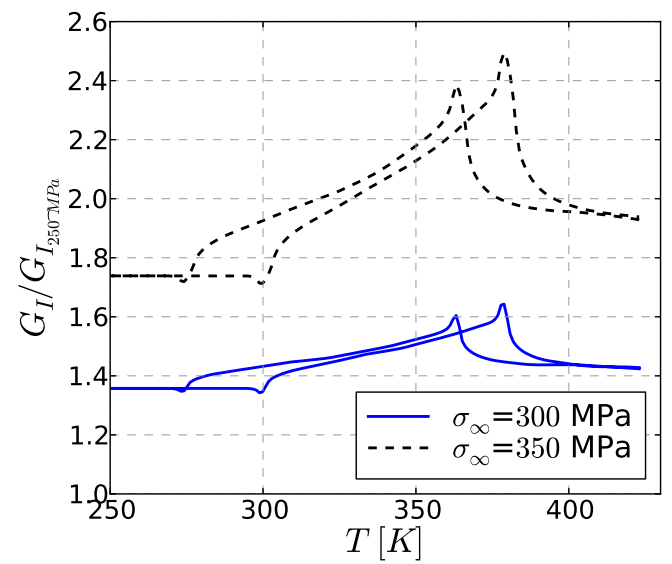

Figure 18: Normalized energy release rate, $G_{I} / G_{I_{250 \mathrm{MPa}}}$, versus temperature $T$, for values of the non-dimensional parameters chosen so as to conform with those of $\mathrm{Ni}_{60} \mathrm{Ti}_{40}$ (wt\%) (Table 1). $G_{I_{250 \mathrm{MPa}}}$ is the energy release rate for $\sigma_{\infty}=250 \mathrm{MPa}$ during thermal cycle.

Table 1: Parameter values used for the numerical results presented in Figure 18. These values correspond to $\mathrm{Ni}_{60} \mathrm{Ti}_{40}$ (wt\%) [48].

\begin{tabular}{lr|lr}
\hline parameter & value & parameter & value \\
\hline$E_{A}[\mathrm{MPa}]$ & 75150 & $H_{\text {sat }}$ & 0.0135 \\
$E_{M}[\mathrm{MPa}]$ & 51000 & $k\left[\mathrm{MPa}^{-1}\right]$ & 0.0022 \\
$\nu_{A}=\nu_{M}$ & 0.33 & $M_{f}[\mathrm{~K}]$ & 268 \\
& & $M_{s}[\mathrm{~K}]$ & 357 \\
& & $A_{s}[\mathrm{~K}]$ & 293 \\
& & $A_{f}[\mathrm{~K}]$ & 372 \\
& & $C_{A}\left[\mathrm{MPa} \mathrm{K} K^{-1}\right]$ & 22.16 \\
& & $C_{M}\left[\mathrm{MPa} \mathrm{K}^{-1}\right]$ & 23.55 \\
& & $n_{1}, n_{2}, n_{3}, n_{4}$ & $0.18,0.25,0.18,0.18$ \\
\hline
\end{tabular}

material parameters, it is instructive to examine the influence of $\sigma_{\infty}$ on the energy release rate (Figure 18). The numerical calculations in Figure 18 are for values of dimensional parameters chosen so as to conform with those of $\mathrm{Ni}_{60} \mathrm{Ti}_{40}(\mathrm{wt} \%)$ and yield quantitative results on the energy release rate values expected for this specific material during actuation. As $\sigma_{\infty}$ increases, the values of the non-dimensional parameters $E_{A} H_{s a t} / \sigma_{\infty}, C_{M}\left(A_{s}-M_{s}\right) / \sigma_{\infty}, C_{M}\left(A_{f}-\right.$ $\left.A_{s}\right) / \sigma_{\infty}, C_{M}\left(M_{s}-M_{f} / \sigma_{\infty}, C_{M} / C_{A}\right.$ decrease while the value of $k \sigma_{\infty}$ increases. Since the influence of decreasing values of $E_{A} H_{s a t} / \sigma_{\infty}, C_{M}\left(A_{s}-\right.$ 
$\left.M_{s}\right) / \sigma_{\infty}, C_{M}\left(A_{f}-A_{s}\right) / \sigma_{\infty}, C_{M}\left(M_{s}-M_{f} / \sigma_{\infty}, C_{M} / C_{A}\right.$ and increasing values of $k \sigma_{\infty}$ on the energy release rate is competitive among some of them, the respective influence of the $\sigma_{\infty}$ cannot be inferred directly. As it can be seen in Figure 18, the higher the applied load levels, the higher the enhancement in the $G_{I} / G_{\infty}$-value.

The dependence of energy release rate on the bias load, transformation strain, slopes of the strain-temperature curve during transformation, and mismatch of Young's moduli between the two phases is relatively strong implying that the actual shape of the strain-temperature curve at constant applied load is important for the quantitative determination of the expected increase in the energy release rate during actuation.

\section{Conclusions}

In this work, finite element calculations are carried out to investigate the effect of global thermomechanically-induced phase transformation on the mechanical fields close to the crack tip and the driving force for crack growth in SMAs. The study focuses on the prototype problem of an infinite center-cracked austenitic SMA plate subjected to a thermal cycle under plane strain, mode I, constant applied loading. The constitutive response of the material is taken to be characteristic of polycrystalline SMAs with the constitutive law accounting for the generation and recovery of all martensitic variants in a volume average sense.

Assuming an elastic behavior for martensite, the crack-tip energy release rate is identified as a single parameter sufficient to describe the full range of neartip fracture environments, and therefore qualifies as the driving force for crack growth even under large scale transformation conditions. This assumption, as discussed in the Introduction, may be applicable to SMA materials systems beyond those that show considerable strength against dislocation plasticity. On the contrary, in conventional elastic-plastic materials, single parameter fracture mechanics theories break down in the presence of extensive yielding. Although suggestions have been made that three parameters, the two being a measure of the stress triaxiality and the Lode parameter, suffice to describe the full range of near-tip fracture environments, material fracture under large scale yielding still remains unresolved.

According to the results, during cooling, the crack-tip energy release rate initially increases as a result of martensitic transformation until it reaches a peak and then decreases before attaining a value at the martensitic-finish temperature, $M_{f}$, which remains constant under further cooling. During heating, the energy release rate starts increasing at the austenitic-start temperature, $A_{s}$, at which reverse phase transformation initiates, then, after reaching a peak, it decreases before attaining, eventually, a constant value. The increase of the energy release rate during a thermal cycle at constant applied loading is substantial, an order of magnitude for some material systems, and should be attributed to the stress redistribution caused by large scale phase transformation. Thus, if crack growth is assumed to occur when the crack-tip energy release rate reaches 
a material (martensite) specific critical value, then crack growth may occur due to phase transformation resulting from thermal variations at constant applied loading.

As indicated above, the energy release rate may reach the material specific critical value during cooling under a constant mechanical load and initiate crack growth. The possibility of a fracture toughening response associated with crack advance, i.e., stable crack growth, should be exploited and studied. As already mentioned, a strong geometry dependence of crack growth resistance is expected as the outer boundaries will exert an influence on the crack-tip deformation state in cases of large scale transformation. The present study of actuationinduced fracture in SMAs should therefore be extended to crack configurations and actuation loading paths beyond that of the infinite center-crack problem and constant-stress, respectively, in an effort to reveal the effect of stress triaxiality on the driving force for crack initiation and growth, and fill the gap between the test and working conditions.

\section{Acknowledgments}

This material is based upon work supported by the National Science Foundation under Grant Numbers CMMI-1301139 and DMR-0844082.

\section{References}

[1] D. Hartl, D. Lagoudas, Aerospace applications of shape memory alloys, Proceedings of the Institution of Mechanical Engineers, Part G: Journal of Aerospace Engineering, SAGE, 2007, pp. 535-552.

[2] M. Sreekumar, T. Nagarajan, M. Singaperumal, M. Zoppi, R. Molfino, Critical review of current trends in shape memory alloy actuators for intelligent robots, Ind. Robot 34 (4) (2007) 285-294.

[3] A. Nespoli, S. Besseghini, S. Pittaccio, E. Villa, S. Viscuso, The high potential of shape memory alloys in developing miniature mechanical devices: A review on shape memory alloy mini-actuators, Sensor Actuat. A-Phys. 158 (1) (2010) 149-160.

[4] A. McKelvey, R. Ritchie, Fatigue-crack growth behavior in the superelastic and shape-memory alloy Nitinol, Metall. Mater. Trans. A 32 (2001) 731743.

[5] S. Robertson, A. Metha, A. Pelton, R. Ritchie, Evolution of crack-tip transformation zones in superelastic Nitinol subjected to in situ fatigue: A fracture mechanics and synchrotron X-ray micro-diffraction analysis, Acta Mater. 55 (2007) 6198-6207.

[6] M. Daymond, M. L. Young, J. Almer, D. Dunand, Strain and texture evolution during mechanical loading of a crack tip in martensitic shapememory NiTi, Acta Mat. 55 (2007) 3929-3942. 
[7] S. Gollerthan, M. L. Young, A. Baruj, J. Frenzel, W. Schmahl, G. Eggeler, Fracture mechanics and microstructure in NiTi shape memory alloys, Acta Mater 57 (2009) 1015-1025.

[8] A. Creuziger, L. Bartol, K. Gall, W. Crone, Fracture in single crystal NiTi, J. Mech. Phys. Solids 56 (2008) 2896-2905.

[9] T. Baxevanis, D. Lagoudas, A mode I fracture analysis of a center-cracked infinite shape memory alloy plate under plane stress, Int. J. Fract. 175 (2) (2012) 151-166.

[10] T. Baxevanis, Y. Chemisky, D. Lagoudas, Finite element analysis of the plane strain crack-tip mechanical fields in pseudoelastic shape memory alloys, Smart Mater. Struct. 21, doi: 10.1088/0964-1726/21/9/094012.

[11] T. Baxevanis, A. Parrinello, D. Lagoudas, On the fracture toughness enhancement due to stress-induced phase transformation in shape memory alloys, Int. J. Plast. 50 (2013) 158-169.

[12] T. Baxevanis, C. Landis, D. Lagoudas, On the fracture toughness of pseudoelastic shape memory alloys, ASME J. Appl. Mech. 81 (4), doi: 10.1115/1.4025139.

[13] T. Baxevanis, C. Landis, D. Lagoudas, On the effect of latent heat on the fracture toughness of pseudoelastic shape memory alloys, ASME J. Appl. Mech. 81 (10), doi: 10.1115/1.4028191.

[14] T. Baxevanis, D. C. Lagoudas, Fracture mechanics of shape memory alloys: review and perspectives, Int. J. Fract. 191 (2015) 191-213.

[15] K. Gall, N. Yang, H. Sehitoglu, Y. Chumlyakov, Fracture of precipitated NiTi shape memory alloys, Int. J. Fract. 109 (2001) 189-207.

[16] S. Robertson, A. Pelton, R. Ritchie, Mechanical fatigue and fracture of nitinol, Int. Materi. Rev. 57 (1) (2012) 1-36.

[17] G. Irwin, Linear fracture mechanics, fracture transition, and fracture control, Eng. Fract. Mech. 1 (2) (1968) 241-257.

[18] R. Vaidyanathan, D. Dunand, U. Ramamurty, Fatigue crack-growth in shape-memory NiTi and NiTi-TiC composites, Mater. Sci. Engi. A 289 (12) (2000) 208-216.

[19] S. Robertson, A. Mehta, A. Pelton, R. Ritchie, Evolution of crack-tip transformation zones in superelastic Nitinol subjected to in situ fatigue: A fracture mechanics and synchrotron X-ray microdiffraction analysis, Acta Mater. 55 (18) (2007) 6198-6207. 
[20] S. Robertson, R. Ritchie, In vitro fatigue-crack growth and fracture toughness behavior of thin-walled superelastic nitinol tube for endovascular stents: A basis for defining the effect of crack-like defects, Biomaterials 28 (4) (2007) 700-709.

[21] G. Stam, E. van der Giessen, Effect of reversible phase transformations on crack growth, Mech. Mater. 21 (1995) 51-71.

[22] S. Yi, S. Gao, Fracture toughening mechanism of shape memory alloys due to martensite transformation, Int. J. Solids Struct. 37 (2000) 5315-5327.

[23] S. Yi, S. Gao, S. Shen, Fracture toughening mechanism of shape memory alloys under mixed-mode loading due to martensite transformation, Int. J. Solids Struct. 38 (2001) 4463-4476.

[24] Y. Freed, L. Banks-Sills, Crack growth resistance of shape memory alloys by means of a cohesive zone model, J. Mech. Phys. Solids 55 (2007) 21572180 .

[25] J. Shaw, S. Kyriakides, A phenomenological model for pseudoelasticity of shape memory alloys under multiaxial proportional and nonproportional loadings, J. Mech. Phys. Solids 43 (1) (1995) 1243-1281.

[26] H. Prahlad, I. Chopra, Development of a strain-rate dependent model for uniaxial loading of SMA wires, J. Intell. Mater. Syst. Struct. 14 (14) (2003) 429-442.

[27] Y. Yan, H. Yin, Q. Sun, Y. Huo, Rate dependence of temperature fields and energy dissipations in non-static pseudoelasticity, Continuum Mech. Therm. 24 (4-6) (2012) 675-695.

[28] Y. He, H. Yin, R. Zhou, Q. Sun, Ambient effect on damping peak of NiTi shape memory alloy, Mater. Lett. 64 (13) (2010) 1483-1486.

[29] Y. He, Q. Sun, On non-monotonic rate dependence of stress hysteresis of superelastic shape memory alloy bars, Int. J. Solids Struct. 48 (11-12) (2011) 1688-1695.

[30] H. Yin, Y. Yan, Y. Huo, Q. Sun, Rate dependent damping of single crystal CuAlNi shape memory alloy, Mater. Lett. 109 (2013) 287-290.

[31] J. Boyd, D. Lagoudas, A thermodynamical constitutive model for shape memory materials. Part I: The monolithic shape memory alloy, Int. J. Plast. 12 (1996) 805-842.

[32] D. Lagoudas (Ed.), Shape Memory Alloys: Modelling and Enginnering Applications, Springer, New-York, 2008.

[33] E. Rybicki, M. Kanninen, A finite element calculation of stress intensity factors by a modified crack closure integral, Eng. Fract. Mech. 9 (1977) 931-938. 
[34] R. Krueger, Virtual crack closure technique: History, approach, and applications, Appl. Mech. Rev. 57 (2004) 109-143.

[35] D. Xie, S. Biggers, Progressive crack growth analysis using interface element based on the virtual crack closure technique, Fin. Elem. Anal. Des. 42 (2006) 977-984.

[36] G. Irwin, Handbuch der Physik VI, Spinger Verlag, Berlin, Germany, 1958, Ch. Fracture I, pp. 558-590.

[37] K. Hellan, Introduction to fracture mechanics, McGraw-Hill Inc. US, 1984.

[38] B. Budniansky, J. Hutchinson, J. Lambropoulos, Continuum theory of dilatant transformation toughening in ceramics, Int. J. Solids Struct. 19 (1983) $337-355$.

[39] J. Lambropoulos, Shear, shape and orientation effects in transformation toughening, Int. J. Solids Struct. 22 (1986) 1083-1106.

[40] J. Eshelby, Solid state physics, Academic, New York, 1956, Ch. The continuum theory of lattice defects.

[41] J. Eshelby, Inelastic behavior of solids, McGraw-Hill, New York, 1956, Ch. Energy relations and the energy momentum tensor in continuum mechanics.

[42] J. Rice, A path independent integral and approximate analysis of strain concentration by notches and cracks, ASME J. Appl. Mech. 35 (1968) 379386.

[43] J. Hutchinson, Fundamentals of the phenomenological theory of nonlinear fracture mechanics, ASME J. Appl. Mech. 50 (4B) (1983) 1042-1051.

[44] D. Parks, The virtual crack extension method for nonlinear material behavior, Comp. Methods Appl. Mech. Eng. 12 (1977) 353-364.

[45] F. Li, C. Shih, A. Needleman, A comparison of methods for calculating energy release rates, Eng. Fract. Mech 21 (1985) 405-421.

[46] C. Shih, B. Moran, T. Nakamura, Energy release rate along a threedimensional crack front in a thermally stressed body, Int. J Fract. 30 (1986) 79-102.

[47] D. Carka, C. Landis, On the path-dependence of the $J$-integral near a stationary crack in an elastic-plastic material, ASME J. Appl. Mech. 78, doi: 10.1115/1.4001748.

[48] D. Hartl, D. Lagoudas, F. Calkins, Advanced methods for the analysis, design, and optimization of sma-based aerostructures, Smart Materials and Structures 20 (9), doi: 10.1088/0964-1726/20/9/094006. 